\title{
Vibration Analysis as a Diagnosis Tool for Health Monitoring of Industrial Machines
}

\author{
Arturo Garcia-Perez, ${ }^{1}$ Juan Pablo Amezquita-Sanchez, ${ }^{2}$ \\ Daniel Morinigo-Sotelo, ${ }^{3}$ and Konstantinos N. Gyftakis ${ }^{4}$ \\ ${ }^{1}$ HSPdigital, DICIS, Universidad de Guanajuato, 36885 Salamanca, GTO, Mexico \\ ${ }^{2}$ Faculty of Engineering, Autonomous University of Queretaro, 76806 San Juan del Rio, QRO, Mexico \\ ${ }^{3}$ Department of Electrical Engineering, University of Valladolid, 47011 Valladolid, Spain \\ ${ }^{4}$ School of Computing, Electronics and Mathematics, Faculty of Engineering, Environment and Computing, Coventry University, \\ Coventry CV1 5FB, UK
}

Correspondence should be addressed to Arturo Garcia-Perez; agarcia@hspdigital.org

Received 6 June 2016; Accepted 6 June 2016

Copyright (C) 2016 Arturo Garcia-Perez et al. This is an open access article distributed under the Creative Commons Attribution License, which permits unrestricted use, distribution, and reproduction in any medium, provided the original work is properly cited.

The need for health monitoring in industrial machines using vibration analysis for diagnosis is an ever growing requirement in all types of industries and equipment. Vibrations in machinery can take various forms, and most of the time these vibrations are unintended and undesirable. Therefore, monitoring the vibration characteristics of a machine can provide the information of its health condition, and this piece of information can be used to detect problems that might be incipient or developing.

The regular use of a machine condition monitoring system allows for observing the problems during their incipient stage or when they are developing. Sometimes a machine can be running into a major failure, even though it appears to be functioning normally. This could lead to a dangerous situation because if this faulty condition is not monitored and detected on time, the problem could lead to the manufacturing of poor quality products, large yield losses, rework costs, and so forth. The vibration signature of a specific machine can then be processed to extract the features related to the fault and give a diagnosis of the machine condition.

$\mathrm{H}$. Li et al. propose a theory with experiment to investigate the influence on modal parameters of thin cylindrical shell (TCS) under bolt looseness boundary, where it can be found that bolt looseness boundary can significantly affect frequency and damping results which might be caused by changes of nonlinear stiffness, damping, and position of connecting bolt looseness. S. Fu et al. present a new approach for mechanical fault diagnosis based on time-domain analysis and adaptive fuzzy C-means clustering. By analyzing vibration of the bearing, it could be detected fast and accurately, to estimate whether the rolling bearing is healthy or not, even single fault or multiple faults. J. Zhou et al. deal with a novel modeling method of planetary gear system, in which the Hertz contact theory is induced to describe impact of the gear pairs under the condition of the high speed and light load. The vibroimpact characteristics of the system are analyzed.

A damage detection method based on flexibility change by using single nonfirst mode is addressed by B. Zhao et al., and the results of the numerical examples (on a cantilever beam and a rod-fastened-rotor with different damage scenarios) indicate that the proposed method can effectively identify single, double, and multiple damage. L. Zhang et al. improve the fault detection accuracy for rolling bearings, an automated fault diagnosis system based on lifting wavelet packet transform (LWPT), sample entropy (SampEn), and classifier ensemble. Experiments were conducted on electric motor bearings considering various sets of bearing categories and fault severity levels. J. Rangel-Magdaleno et al. verify an experimental study of partially damaged rotor bar in induction motor under different load conditions based on discrete 
wavelet transform analysis. The approach is based on the extraction of features from vibration signals at different levels of damage and three mechanical load conditions.

An adaptive stochastic resonance and analytical mode decomposition-ensemble empirical mode decomposition (AMD-EEMD) method is proposed for fault diagnosis of rotating machinery by $\mathrm{P}$. Shi et al. Z. K. Abdul et al. have shown that $1 \mathrm{D}$ LPB (local binary pattern) is an effective technique to extract features for detecting fault in gear when data with the same speed and/or load are available in training and testing the model. Moreover, the 1D LBP is cheaper in terms of computation than the 2D LBP scheme. J. J. Saucedo-Dorantes et al. propose a methodology based on vibration analysis and current analysis for the diagnosis of different levels of uniform wear in a gearbox and the detection of bearing defects, both linked in the same kinematic chain. The methodology is based on the acquisition of vibration signals that are generated in the kinematic chain and induction motor current signals.

Besides those, there are several interesting works in the issue. D. A. Elvira-Ortiz et al. suggest a system capable of providing complete joint kinematics and position of an industrial robot and tested in a 6-degree-of-freedom robot. The system is based on KF technique for vibration suppression and for fusing the measurements of two primary sensors: gyroscopes and accelerometers. J. Yao et al. deal with a pulse adaptive time-frequency transform method to extract the fault features of the damaged rolling bearing. An experimental investigation into vibration characteristics of passed and failed lapping scenarios which discusses a potential solution to minimizing the serious damage so-called plate scratch which intermittently occurs in such process is presented by J. Suwatthikul et al. B. Li et al. propose a feature extraction and selection scheme for intelligent engine fault diagnosis by utilizing twodimensional nonnegative matrix factorization, mutual information, and nondominated sorting genetic algorithms, and finally $\mathrm{H}$. Wang perform a two-stage compression method to reduce the large amount of the faulty vibration signals induced by the condition monitoring bearings.

\section{Acknowledgments}

The guest editors would like to thank the authors for their contributions to the special issue and all the reviewers for their constructive reviews. We are also grateful to the Editorin-Chief, Mehdi Ahmadian, for his support.

Arturo Garcia-Perez

Juan Pablo Amezquita-Sanchez

Daniel Morinigo-Sotelo

Konstantinos N. Gyftakis 


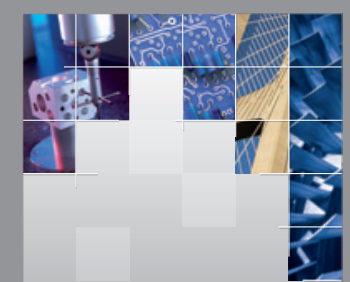

\section{Enfincering}
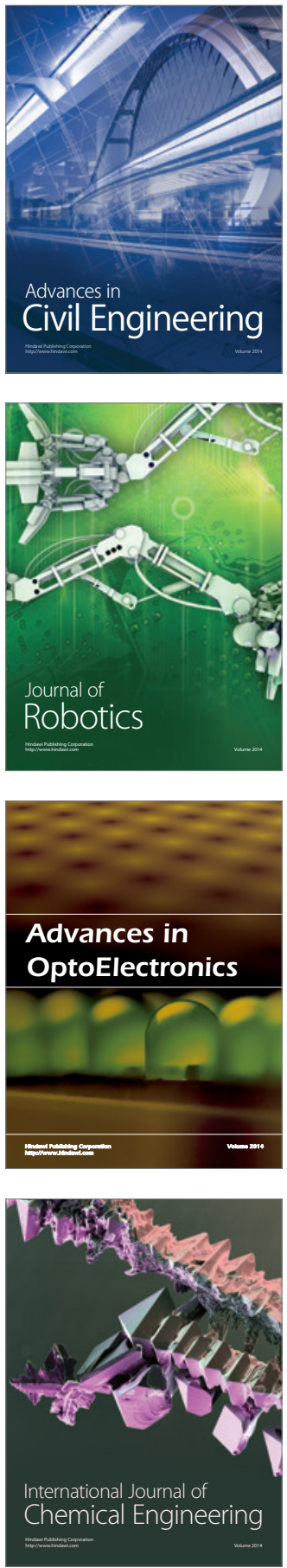

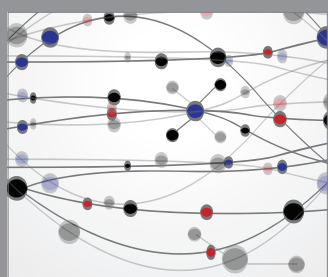

The Scientific World Journal

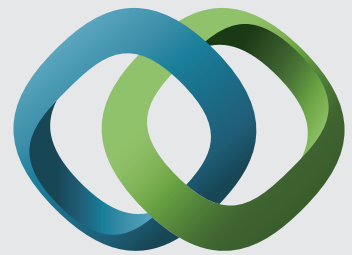

\section{Hindawi}

Submit your manuscripts at

http://www.hindawi.com
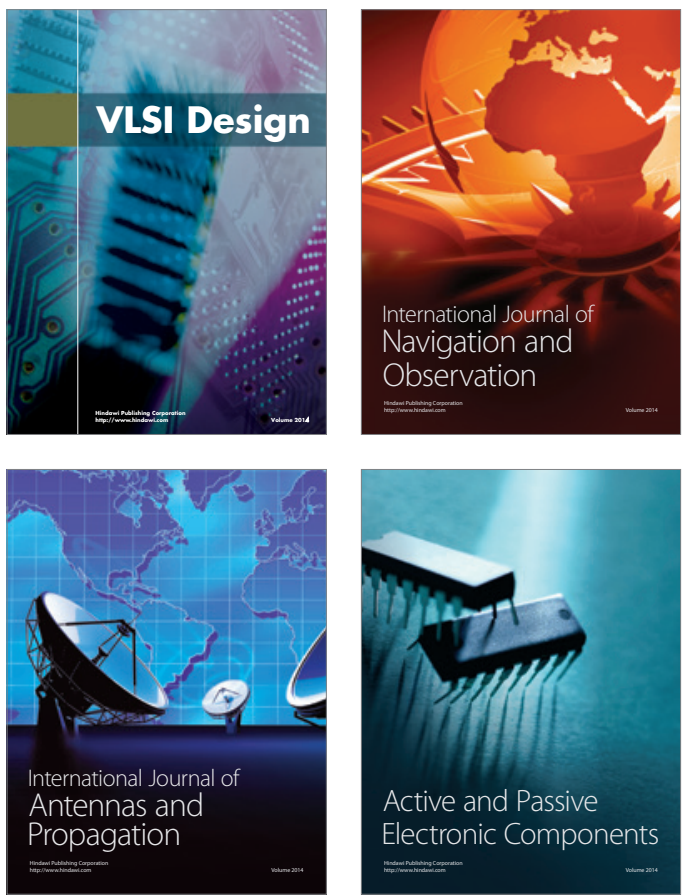
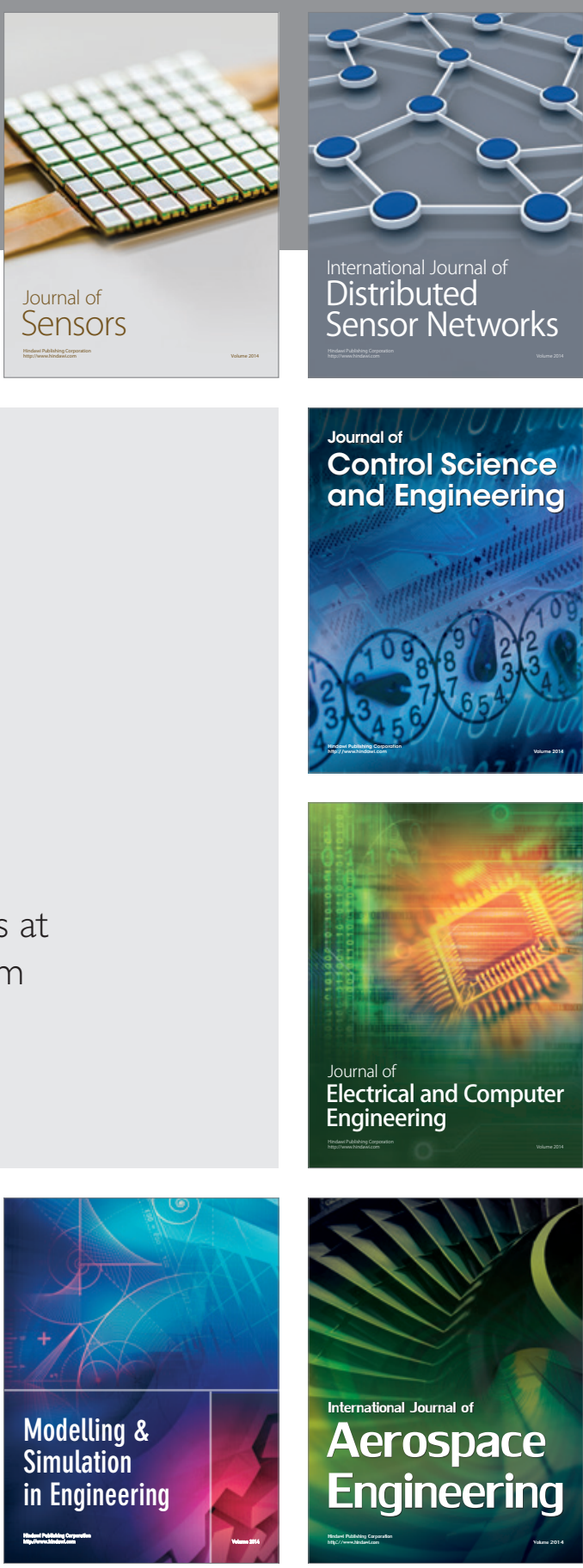

International Journal of

Distributed

Sensor Networks

Journal of

Control Science

and Engineering
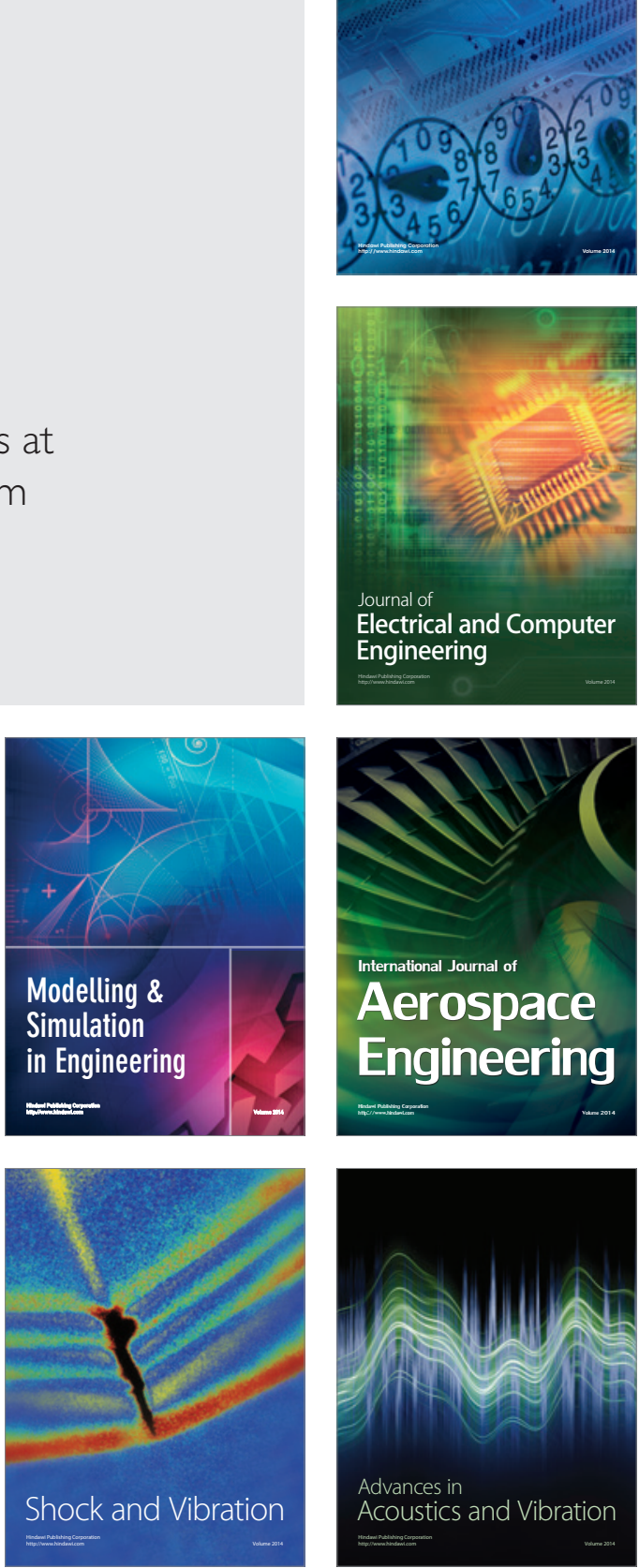